\title{
TNF superfamily member TWEAK exacerbates inflammation and demyelination in the cuprizone-induced model
}

\author{
Heather A. Iocca ${ }^{a}$, Sheila R. Plant ${ }^{\mathrm{a}, \mathrm{b}, 1}$, Ying Wang ${ }^{\mathrm{a}}$, Laura Runkel ${ }^{\mathrm{c}, 2}$, Brian P. O'Connor ${ }^{\mathrm{a}}$, \\ Emma T. Lundsmith ${ }^{a}$, Kyungmin Hahm ${ }^{\mathrm{c}}$, Hendrik W. van Deventer ${ }^{\mathrm{a}, \mathrm{d}}$, \\ Linda C. Burkly ${ }^{\mathrm{c}, *}$, Jenny P-Y. Ting ${ }^{\mathrm{a}, \mathrm{b}, \mathrm{e}, *}$ \\ ${ }^{a}$ Lineberger Comprehensive Cancer Center, University of North Carolina at Chapel Hill, NC 27599-7295, United States \\ ${ }^{\mathrm{b}}$ Neuroscience Center, University of North Carolina at Chapel Hill, NC 27599-7295, United States \\ ${ }^{\mathrm{c}}$ Molecular Discovery, Biogen Idec, 12 Cambridge Center, Cambridge, MA 02142, United States \\ d Division of Hematology/Oncology, Department of Medicine, University of North Carolina at Chapel Hill, NC 27599-7295, United States \\ ${ }^{\mathrm{e}}$ Department of Microbiology and Immunology, University of North Carolina at Chapel Hill, NC 27599-7295, United States
}

Received 28 August 2007; received in revised form 30 November 2007; accepted 3 December 2007

\begin{abstract}
Inflammatory cytokines have been implicated in the pathology of multiple neurologic diseases, including multiple sclerosis. We examined the role of the TNF family member TWEAK in neuroinflammation. Cuprizone-fed mice undergo neuroinflammation and demyelination in the brain, but upon removal of cuprizone from the diet, inflammation is resolved and remyelination occurs. Using this model, we demonstrate that mice lacking TWEAK exhibit a significant delay in demyelination and microglial infiltration. During remyelination, mice lacking the TWEAK gene demonstrate only a marginal delay in remyelination. Thus, this study identifies a primary role of TWEAK in promoting neuroinflammation and exacerbating demyelination during cuprizone-induced damage.
\end{abstract}

(C) 2007 Elsevier B.V. All rights reserved.

Keywords: Multiple sclerosis; Neuroinflammation; CNS; Cuprizone

\section{Introduction}

Central nervous system (CNS) inflammation is an important component of demyelinating disease. The most common demyelinating disorder, multiple sclerosis (MS), is mediated by both immune cell infiltration and inflammatory cytokine

\footnotetext{
* Corresponding authors. J.P.-Y. Ting is to be contacted at Lineberger Comprehensive Cancer Center, CB\#7295, University of North Carolina, Chapel Hill, NC 27599, United States. Tel.: +1 919966 5538; fax: +1 9199668212. L.C. Burkly, Molecular Discovery, Biogen Idec, 12 Cambridge Center, Cambridge, MA 02142, United States. Tel.: +1 617679 3329; fax: +1 617 6793148.

E-mail addresses: linda.burkly@biogenidec.com (L.C. Burkly), panyun@med.unc.edu (J.P.-Y. Ting).

${ }^{1}$ Current affiliation: Division of Neurology, Department of Medicine, Duke University, Durham, NC, 27710, United States.

${ }^{2}$ Current affiliation: Citeline, Inc. 13456 Princess Margaret Dr., Jacksonville, FL, United States.
}

production. Although inflammatory cytokines have traditionally been thought to contribute to the destruction of myelin in the CNS, recent evidence suggests that inflammation and inflammatory mediators may also be important contributors to CNS repair (Liu et al., 1998; Arnett et al., 2001; Kassiotis and Kollias, 2001). The inflammatory process is an attractive target for potential therapy but care must be taken to ensure that such targets do not adversely affect repair processes.

The cuprizone model provides an ideal system to study the processes of neuroinflammation, demyelination and remyelination (Suzuki and Kikkawa, 1969; Blakemore, 1972, 1973a,b; Arnett et al., 2001; Plant et al., 2005, 2006). Cuprizone, a copper chelating agent, results in a predictable course of oligodendrocyte cell death and microglial infiltration at the site of damage in the murine CNS, particularly the midline corpus callosum. In contrast to the experimental autoimmune encephalomyelitis (EAE) model, where demyelination is dependent 
on CD4+ T cells, demyelination in the cuprizone model is mediated primarily by resident microglia with a small contribution from peripheral macrophages (McMahon et al., 2002). Although considerable attention has been paid in the literature to the role of $\mathrm{CD} 4+\mathrm{T}$ cells in MS, evidence exists to suggest that some inflammatory lesions occur in the absence of $\mathrm{T}$ cell involvement (Lucchinetti et al., 2000). The lesions observed during cuprizone treatment mimic the type III and type IV lesions observed in the brains of MS patients (Lucchinetti et al., 2000). Thus, understanding the inflammatory response in the cuprizone model may contribute to the elucidation of the role of the inflammatory response in disease progression and thereby address CNS pathology caused by this mechanism. Another major attribute of this model is that cuprizone treatment results in a predictable time course and pattern of demyelination and remyelination enabling the study of both phases of disease.

The tumor necrosis factor (TNF) superfamily comprises greater than 40 signaling molecules and receptors (Hehlgans and Pfeffer, 2005). Despite structural similarities, each molecule within the TNF superfamily exhibits distinct, pleiotrophic, context-dependent effects. The prototypical TNF family member, TNF $\alpha$, has been successfully targeted for treatment of autoimmune and inflammatory diseases, most notably inhibition of TNF $\alpha$ for the treatment of rheumatoid arthritis and Crohn's disease (Knight et al., 1993; Mohler et al., 1993; Targan et al., 1997; D'Haens et al., 2001). TNF $\alpha$ exists in both membrane bound (memTNF) and soluble forms. Cleavage of memTNF by TNF $\alpha$-converting enzyme (TACE) results in release of the soluble molecule (Black et al., 1997). TNF $\alpha$ signals through two cell surface receptors, TNF receptors 1 (TNFR1) and 2 (TNFR2) (Brockhaus et al., 1990). TNFR1 contains an intracellular death domain and is expressed by most cell types; TNFR2 lacks a death domain and is expressed by a restricted subpopulation. Increasing evidence suggests that TNFR2 may function in reparative processes (Akerman et al., 1992; Streit et al., 1998; Ruuls et al., 2001). TNFR2 is implicated as the major receptor for memTNF signaling (Grell et al., 1999).

TNF-like weak inducer of apoptosis (TWEAK) (TNFSF12, Apo3L) is a relatively recently identified member of the TNF superfamily (Chicheportiche et al., 1997). Similar to many other members, TWEAK is synthesized as a $30 \mathrm{kDa}$ type II transmembrane protein, is cleaved to its soluble $19 \mathrm{kDa}$ form and signals as a trimerized molecule (Chicheportiche et al., 1997). TWEAK signaling occurs through its only known cell surface receptor Fn14 (TNFRSF12A, CD266) (Nakayama et al., 2003; Wiley and Winkles, 2003; Brown et al., 2006) which lacks a death domain and binds TNF receptor associated factors (TRAFs) 1, 2, 3, and 5 (Brown et al., 2003). Like other family members, TWEAK/Fn14 signaling also mediates context-dependent pleiotropic effects, with potential results including cell death (Chicheportiche et al., 1997; Nakayama et al., 2002; Nakayama et al., 2003), cell proliferation (Desplat-Jego et al., 2002; Jakubowski et al., 2005; Michaelson et al., 2005; Perper et al., 2006), pro-angiogenic and/or pro-inflammatory activity via NFKB-driven cytokine and chemokine secretion and upregulation of ICAM (Campbell et al., 2004; Campbell et al., 2006). Significant evidence exists for a role for TWEAK in autoimmune disorders (Chicheportiche et al., 2000). TWEAK is encoded on human chromosome 17 p13 (Chicheportiche et al., 1997), which corresponds to a syntenic region in mice that contains candidate genes for the lupus-like autoimmune diseases. Furthermore, lupus patients and mice with lupus-like autoimmune diseases exhibit aberrant TWEAK expression (Chicheportiche et al., 2000; Kaplan et al., 2002). TWEAK is dramatically upregulated in a murine collagen-induced arthritis model and anti-TWEAK neutralizing monoclonal antibodies significantly reduce disease severity (Perper et al., 2006). Studies of TWEAK expression in the CNS have indicated that TWEAK is expressed primarily by astrocytes and microglia (Desplat-Jego et al., 2002) and is significantly upregulated in the CNS in EAE (Desplat-Jego et al., 2005). Furthermore, when subjected to EAE, TWEAKoverexpressing transgenic mice exhibit exacerbation and higher mean clinical scores than wildtype animals (DesplatJego et al., 2002). Conversely, anti-TWEAK neutralizing monoclonal antibodies reduce the clinical severity of EAE (Desplat-Jego et al., 2005).

Several members of the TNF superfamily have been implicated in the pathology of MS. TNF $\alpha$ and TNF family members including lymphotoxin $\alpha(\mathrm{Lt} \alpha)$ are increased in EAE mice and MS plaques (Ruddle et al., 1990; Woodroofe and Cuzner, 1993; Matusevicius et al., 1996; Navikas et al., 1996; Korner et al., 1997; Liu et al., 1998; Probert et al., 2000; Arnett et al., 2001; Kassiotis and Kollias, 2001). Studies in EAE suggest an exacerbatory role for TNF $\alpha$ in inflammation and disease progression (Ruddle et al., 1990; Baker et al., 1994; Klinkert et al., 1997; Probert et al., 2000). However, a clinical trial designed to inhibit TNF $\alpha$ signaling in patients was halted due to exacerbation of symptoms (van Oosten et al., 1996; Lenercept, 1999; Robinson et al., 2001; Sicotte and Voskuhl, 2001). It was subsequently demonstrated in murine MS models that TNF $\alpha$ may promote repair and immunosuppression as well as damage (Liu et al., 1998; Arnett et al., 2001; Kassiotis and Kollias, 2001). In our study of the cuprizone model, we found that TNF $\alpha$ has a modest exacerbatory effect on demyelination, but dramatically enhances remyelination via its interaction with TNFR2 to increase the proliferation of oligodendrocyte precursors (Arnett et al., 2001). We have further demonstrated that $\mathrm{Lt} \alpha$ exacerbates inflammation and demyelination, as $\mathrm{Lt}^{-/-}$ mice exhibit delayed demyelination but normal remyelination (Plant et al., 2005). Similar effects on demyelination were observed for mice lacking $L t \beta R$, which functions as a receptor for Lt $\alpha / \beta$ heterodimers (Plant et al., 2007). The latter experiments suggest that while blockade of TNF $\alpha$ might produce opposing beneficial and damaging effects on the myelination process, disrupting Lt $\alpha$ and/or Lt $\beta R$ might only reduce demyelination without impairing remyelination. Thus far, studies exploring the role of TWEAK in neuroinflammation have employed CD4+ T cell-dependent EAE (Desplat-Jego et al., 2002, 2005) and the role of TWEAK in remyelination has not been investigated. In the current study, we addressed these issues using the cuprizone model of demyelination and remyelination in $\mathrm{TWEAK}^{-1-}$ mice. 


\section{Materials and methods}

\subsection{Cuprizone treatment of animals}

Male TWEAK ${ }^{-/-}$(backcrossed 7 generations onto $\mathrm{C} 57 \mathrm{Bl} / 6$ ) and corresponding wildtype control mice were bred at Biogen Idec (Cambridge, MA) under an Institutional Animal Care and Use Committee (IACUC) approved protocol. Animals were maintained in the UNC animal facilities in accordance with $\mathrm{NIH}$ guidelines. Protocols were approved by IACUC at UNC, Chapel Hill. All animals were 8-10 weeks of age at the start of cuprizone treatment. At this time, male wildtype and $\mathrm{TWEAK}^{-/-}$mice were fed $0.2 \%$ cuprizone [oxalic bis(cyclohexylindenehydrazide)] (Sigma, St. Louis, MO) in ground chow ad libitum. For demyelination studies mice were treated with cuprizone for 3, $3.5,4$ or 5 weeks. For remyelination studies, mice were treated with cuprizone for 6 weeks and subsequently returned to normal chow for an additional $1,2,4,6$ or 8 weeks $(7,8,10,12$ or 14 weeks of total treatment). Untreated mice were maintained on a diet of normal chow for the duration of the study. A minimum of 10 and maximum of 12 mice were examined for each time point.

\subsection{Quantitative realtime $P C R$}

Total RNA was isolated from a region of dissected brain containing the corpus callosum of wildtype mice at $0,3,4,5,7,8$, or 10 weeks of cuprizone treatment using the Qiagen RNeasy kit (Qiagen, Valencia, CA). SybrGreen quantitative realtime PCR was performed using an ABI Prism 7900 sequence-detection system (PE Applied Biosystems, Foster City, CA). A $10 \mu \mathrm{L}$ reaction containing $10 \mathrm{nM}$ target primers and Marsh Universal Quantitative PCR Master Mix was used. Intron spanning TWEAK and Fn14 primers were as follows: TWEAK forward 5'-CGA GCT ATT GCA GCC CAT TA-3', TWEAK reverse 5'CCT GCT TGT GCT CCA TCC T-3', Fn14 forward 5'-GAC CTC GAC AAG TGC ATG GA-3', Fn14 reverse 5'-CGC ATC CCA GGC AGA AGT-3'. Primers for cyclophilin expression were as follows: forward 5'-AGG TCC TGG CAT CTT GTC CAT-3', reverse 5'-GAA CCG TTT GTG TTT GGT CCA-3'. Reactions for cyclophilin were performed in parallel with TWEAK and Fn14 reactions to control for equal cDNA input. Thermal cycle parameters were $95^{\circ} \mathrm{C}$ for $10 \mathrm{~min}, 95^{\circ} \mathrm{C}$ for $15 \mathrm{~s}$, $60{ }^{\circ} \mathrm{C}$ for $1 \mathrm{~min}$ for 40 cycles. $p$-values were obtained for individual experiments using Student's $t$ tests. A minimum of 4 experiments were performed and representative results are shown.

\subsection{Tissue preparation and histological analysis}

Mice were deeply anesthetized and perfused sequentially via intra-cardial puncture with phosphate buffered saline (PBS) and $4 \%$ paraformaldehyde (PFA). Brains were removed, post-fixed in $4 \%$ PFA overnight at $4{ }^{\circ} \mathrm{C}$ and paraffin embedded. $5 \mu \mathrm{M}$ coronal brain sections were cut from the fornix region of the corpus callosum as previously described (Arnett et al., 2001). Subsequent histological analyses were limited to the midline corpus callosum. To examine the extent of demyelination and remyelination in the brains of cuprizone treated mice, paraffin sections were rehydrated through graded alcohols and stained with Luxol Fast BluePeriodic Acid Schiff(LFB-PAS, Sigma, St. Louis, MO). Sections were scored on a scale of 0 (complete myelination) to 3 (complete demyelination) by three double-blinded readers as previously described (Arnett et al., 2001; Plant et al., 2005). p-values were obtained with Mann-Whitney non-parametric statistical analysis.

\subsection{Immunohistochemistry}

Sections were deparaffinized and rehydrated through graded alcohols to PBS. Detection of microglia/macrophages, astrocytes, and mature oligodendrocytes was performed using immunohistochemistry as previously described (Plant et al., 2005). To detect microglia, sections were stained with biotinylated RCA-1 (Vector Laboratories, Burlingame, CA) (1:500) and subsequently stained with AlexaFlor 594 - Avidin D (Molecular Probes, Carlsbad, CA) (1:500). RCA-1 immunopositive cells with a DAPI-stained nucleus were quantified within a $0.033 \mathrm{~mm}^{2}$ area of the midline corpus callosum. Cell counts represent the average number of positive cells for a minimum of 10 and a maximum of 12 mice per time point. Astrogliosis was observed throughout the corpus callosum/ fornix region of treated mice. Sections were stained for glial fibrillary acidic protein (GFAP) (Dako, Carpinteria, CA) (1:100) followed by incubation with fluorescein-conjugated goat antirabbit secondary antibody (Vector Laboratories) (1:100). Due to challenges quantifying this population, stained sections were scored for degree of astrogliosis on a scale of 0 (normal astrocyte number) to 3 (severe astrogliosis). MBP was detected using antiMBP antibody (1:1000) (Sternberger Monoclonals Inc, Lutherville, MD) followed by fluorescein-conjugated anti-mouse IgG (Invitrogen) (1:1000). MBP positive area was quantitated using MetaMorph v5.0 (Universal Imaging, Sunnyvale, CA) and expressed as percent positive for MBP staining relative to total image size. To detect mature oligodendrocytes, sections were incubated with anti-GST $\pi$ (Stressgen) $(1: 500)$ overnight at $4{ }^{\circ} \mathrm{C}$. Sections were subsequently incubated with biotinylated goat anti-rabbit (1:100, Vector Labs) and Texas red conjugated avidin D (1:500, Vector Labs). All staining was visualized using standard florescence microscopy techniques.

\subsection{Statistical analyses}

Unpaired Student's $t$ tests were used to evaluate data for statistically significant changes between experimental groups unless otherwise indicated. For analysis of demyelination and remyelination by LFB, scores of 3 double-blinded readers were analyzed using Mann-Whitney non-parametric tests. Data are expressed as mean $+/-$ S.E.M. unless otherwise noted.

\section{Results}

3.1. TWEAK and Fn14 RNA expression are increased during the demyelination and remyelination phases of the cuprizone model

Previous reports indicate that TWEAK and/or Fn14 are upregulated during inflammatory processes. However, the 
expression patterns of TWEAK and Fn14 in cuprizone-induced neuroinflammation remain uncharacterized. To determine the expression patterns of TWEAK and Fn14 during cuprizone treatment, RNA was isolated from the brains of wildtype mice after $0,3,4$, or 5 weeks of cuprizone and analyzed by quantitative realtime PCR. To control for equal cDNA input, results were normalized to cyclophilin expression and expressed as fold induction relative to RNA isolated from untreated control animals. TWEAK expression remained relatively stable with a statistically significant but modest increase observed only at the 5 week $(p<0.05)$ time point (Fig. 1A). A statistically significant increase in Fn14 expression was observed at 3, 4, and 5 weeks of cuprizone treatment $(p<0.05)$ with a maximal induction of nearly 2-fold (Fig. 1B).

To determine if TWEAK mRNA expression is altered during remyelination, wildtype mice were treated with cuprizone for 6 weeks and then returned to normal chow for 1,2 , or 4 weeks ( 7,8 or 10 weeks total treatment). RNA was isolated from brain and analyzed by quantitative realtime PCR. All samples were normalized to cyclophilin expression. Expression of TWEAK remained elevated relative to untreated control mice at the 7 week time point $(p=0.013)$ and returned to levels not significantly different from untreated control animals thereafter. Fn14 levels were reduced to levels not significantly elevated

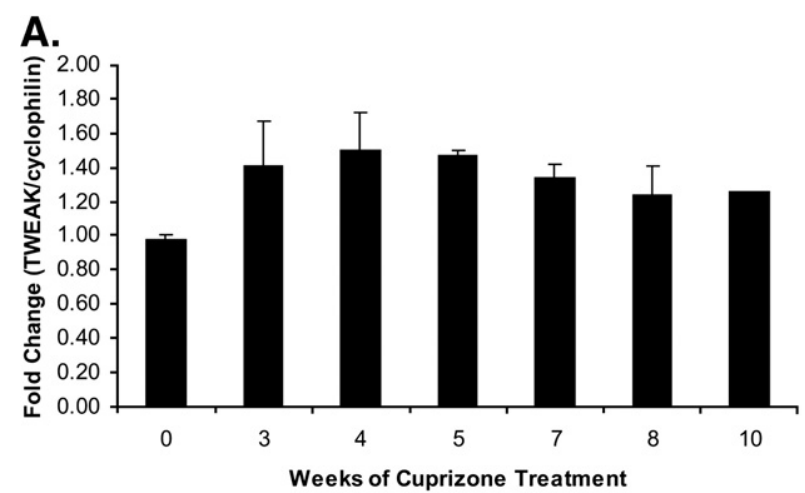

B.

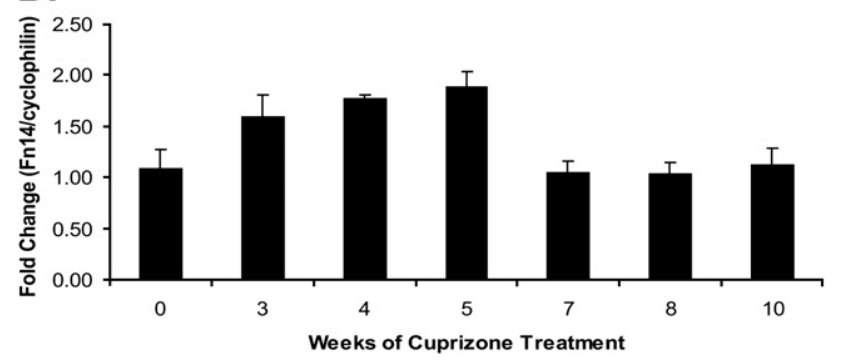

Fig. 1. TWEAK and Fn14 mRNA expression in cuprizone treated mice. RNA was isolated from brains of mice treated with cuprizone for $0,3,4$ or 5 weeks or mice treated with cuprizone for 6 weeks and allowed to recover for 1,2 or 4 weeks ( 7,8 or 10 weeks total treatment). RNA was analyzed by realtime PCR using gene specific primers against TWEAK (A) or Fn14 (B). TWEAK expression was significantly increased at 5 and 7 weeks $(p<0.05)$ before returning to levels not significantly increased relative to baseline. Fn14 expression was significantly increased relative to control $(p<0.05)$ at 3,4 , and 5 weeks. To control for equal RNA input, results were normalized to cyclophilin expression. Data is expressed as fold induction in TWEAK or Fn14 expression relative to control. Graphs indicate representative results of a minimum of 4 experiments. above baseline during all remyelination time points. Thus, modest upregulation of Fn14 and TWEAK occurs during the demyelination phase of cuprizone treatment. It is unclear whether the statistically significant increase in mRNA expression translates to an increase in protein expression and is biologically significant. However, due to the soluble nature of TWEAK and the lack of good reagents for the detection of murine Fn14 by immunohistochemistry, protein expression of these molecules was not assessed. Reagents for the detection of Fn14 by flow cytometry do exist. However, flow cytometry of the adult brain is exceedingly difficult and we were unable to detect Fn14 expression using this technique.

\subsection{TWEAK exerts effects on demyelination and remyelination rates in the cuprizone model}

To elucidate the role of TWEAK in neuroinflammatory processes and demyelination, wildtype and $\mathrm{TWEAK}^{-/}$mice were treated with $0.2 \%$ cuprizone in ground chow for $0,3,3.5$, 4 , or 5 weeks. TWEAK $^{-1-}$ mice exhibited a marked delay in demyelination of the midline corpus callosum relative to wildtype animals as determined by LFB-PAS staining at 3.5 and 4 weeks of treatment (Fig. 2A). This delay was significant at 4 weeks ( $p=0.044$ ) (Fig. 2B). Thus, these results demonstrate that TWEAK exacerbates demyelination. Similar effects on demyelination have been observed for the TNF family members $\mathrm{TNF} \alpha$, Lt $\alpha$ and Lt $\beta \mathrm{R}$ (Arnett et al., 2001; Plant et al., 2005, 2007). As described for other TNF family members, both wildtype and TWEAK ${ }^{-/}$mice were fully demyelinated by the 5 week time point in the face of persistent cuprizone insult.

One of the advantages of the cuprizone model of neuroinflammation is that it enables the study of remyelination as well as demyelination. Following the withdrawal of cuprizone from the diet, mice remyelinate over a predictable time course. To assess the role of TWEAK in remyelination, TWEAK $^{-/-}$and wildtype mice were treated with $0.2 \%$ cuprizone in ground chow for 6 weeks and then returned to normal chow for an additional 1, 2, 4, 6 or 8 weeks to allow remyelination of the CNS $(7,8,10,12$ or 14 weeks of total treatment) (Fig. 2). TWEAK $^{-1-}$ mice remyelinated comparably to wildtype mice through 10 weeks of total treatment (6 weeks of treatment, 4 weeks of recovery) as measured by LFB-PAS staining. A modest but significant delay in remyelination of $\mathrm{TWEAK}^{-/-}$ mice was observed at the 12 week time point ( 6 weeks of treatment, 6 weeks of recovery) $(p=0.019)$. However, it is important to note that by 14 weeks, the extent of remyelination in TWEAK ${ }^{-/-}$and wildtype mice is not significantly different. Thus, although $\mathrm{TWEAK}^{-/-}$mice exhibit a transient delay in remyelination, it is insufficient to result in prolonged or severe remyelination deficiencies in the CNS. This effect is very different from that observed in TNF $\alpha$ null mice in which there is a dramatic and prolonged defect in remyelination (Arnett et al., 2001).

In order to provide a quantitative measurement for the loss of myelin fibers and to confirm LFB data indicating that TWEAK exerts an exacerbatory role in demyelination immunohistochemical staining for myelin basic protein (MBP) was 
A

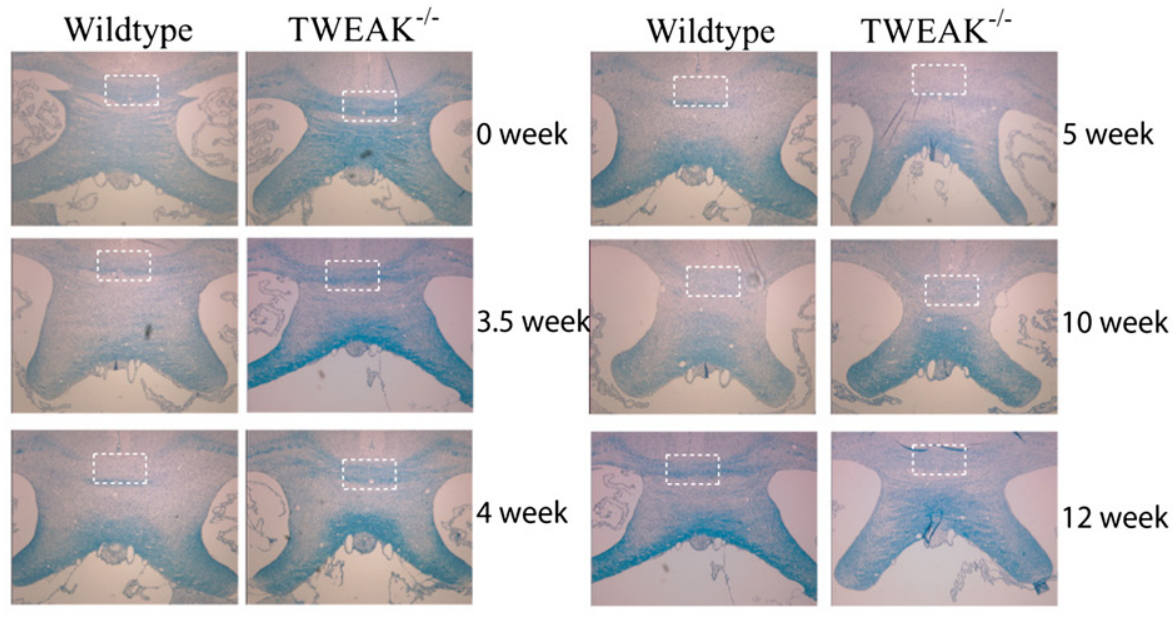

B.
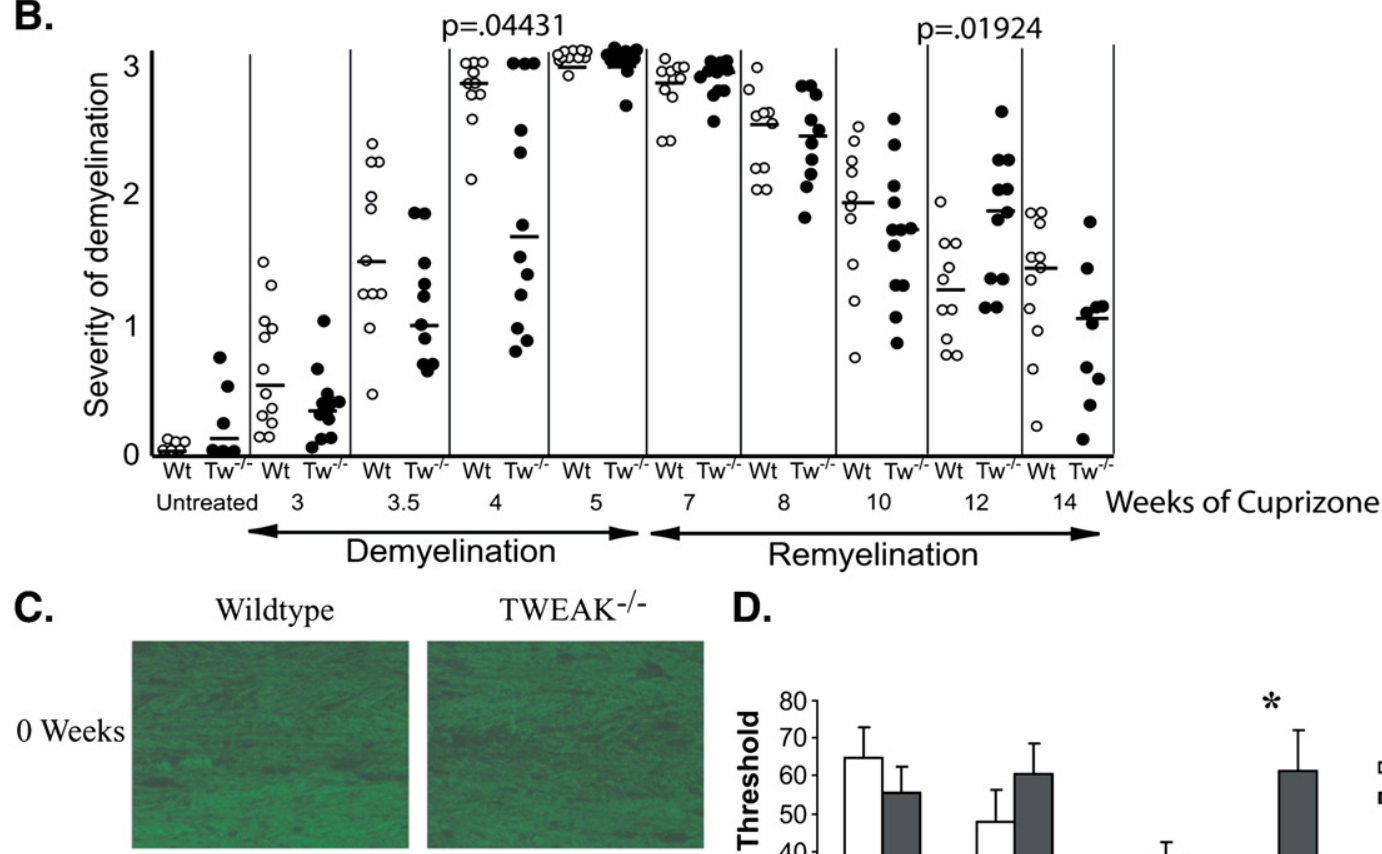

TWEAK $^{-/-}$

D.
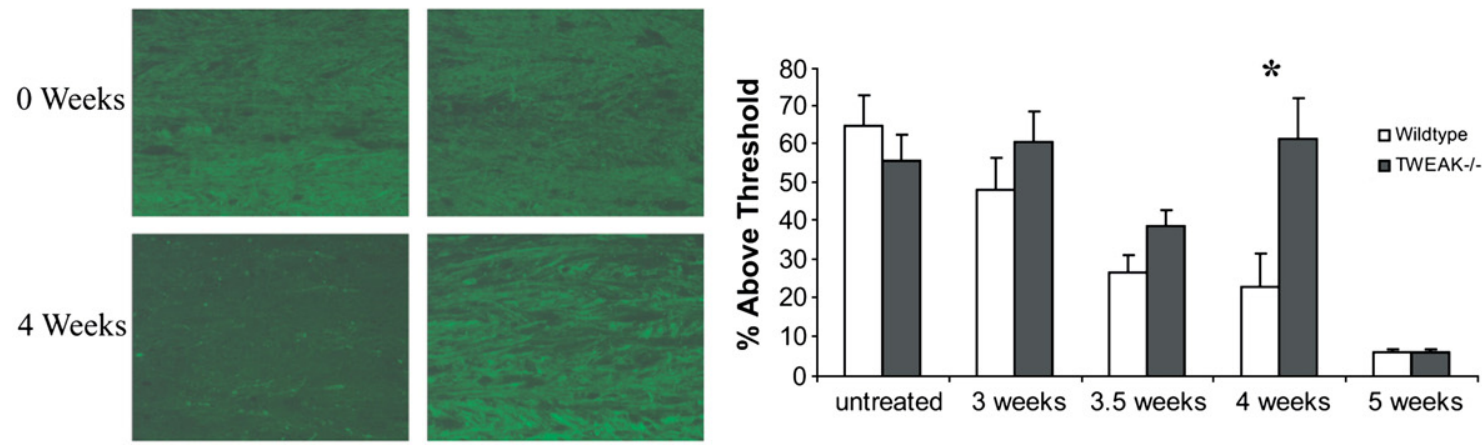

Fig. 2. Time course of demyelination and remyelination in wildtype and TWEAK ${ }^{-/}$mice. (A) LFB-PAS stained paraffin sections were graded at the midline corpus callosum on a scale of 0 (normal) to 3 (fully demyelinated) by 3 double-blinded readers. Dashed box denotes the region of interest. Representative images for $0,3.5,4$, 5,10 and 12 weeks of treatment are shown. (B) Graphical representation of demyelination and remyelination in wildtype and TWEAK ${ }^{-/-}$mice. Each circle represents the average of 3 scores for one mouse. Wildtype mice are denoted by open circles, TWEAK ${ }^{-/-}$mice are denoted by filled circles. Horizontal lines denote the median LFB score for each treatment group. Significant differences in extent of myelination were observed between wildtype and TWEAK ${ }^{-/-}$mice at 4 weeks $(p=0.04431)$ and 12 weeks (0.01924). (C, D) Quantitation of myelin basic protein expression in wildtype and TWEAK ${ }^{-/-}$mice. Paraffin sections of wildtype and TWEAK $^{-/-}$mice $^{-}$ were stained with antibody to myelin basic protein. MBP positive area was quantitated using MetaMorph v5.0 and expressed as percent positive for MBP staining relative to total image size. (C) Representative image of MBP stained paraffin sections at 0 and 4 weeks of cuprizone treatment. (D) Percentage of image positive for MBP relative to total image size. ${ }^{*}$ denotes conditions in which a significant difference exists between wildtype and TWEAK $^{-/-}$mice $(p=0.01)$.

performed. MBP staining revealed a loss of myelin fibers at 4 week time point in wildtype mice (Fig. 2C). Images of MBP immunohistochemistry at midline corpus callosum were obtained, MBP positive area quantitated using MetaMorph v5.0 and results expressed as percentage positive for MBP relative to total image size (Fig. 2D). Overall levels of MBP declined over time out to 5 weeks as described in LFB-PAS studies (compare Fig. 2B and D). A significant difference in mean relative fluorescence intensity was observed between wildtype and $\mathrm{TWEAK}^{-/-}$mice after 4 weeks of cuprizone treatment $(p=0.01)$ demonstrating a delay in loss of myelin fibers in the TWEAK ${ }^{-1-}$ mice. Thus, immunohistochemistry for 
MBP supports the data previously obtained using LFB-PAS staining.

\subsection{TWEAK affects the microglial accumulation but not astrogliosis during demyelination}

The cuprizone model induces neuroinflammation mediated primarily by microglia and macrophage infiltration at the site of CNS injury. To determine the role of TWEAK in microglia/ macrophage infiltration, paraffin sections of wildtype and TWEAK $^{-1-}$ mice were stained with the lectin RCA-1 (10-12 mice/time point) (Suppl. Fig. 1). The number of infiltrating cells in the midline corpus callosum was calculated and represented graphically (Fig. 3A). A statistically significant although moderate reduction in the number of infiltrating microglia and macrophages in TWEAK $^{-/-}$mice was observed after 4 weeks of cuprizone treatment $(p=0.02)$. These data suggest that TWEAK enhances the accumulation of microglia/macrophages at the site of inflammation. This difference disappeared by 5 weeks of cuprizone treatment, indicating that this difference represents a delay in, not a loss of, macrophage/microglia accumulation. In addition to the use of RCA-1 to detect microglia and macrophages infiltrating the CNS, PAS scores were obtained for each LFB-PAS stained section. PAS staining indicates the extent of infiltrating cells in the corpus callosum, although this cellularity is not specifically limited to microglia
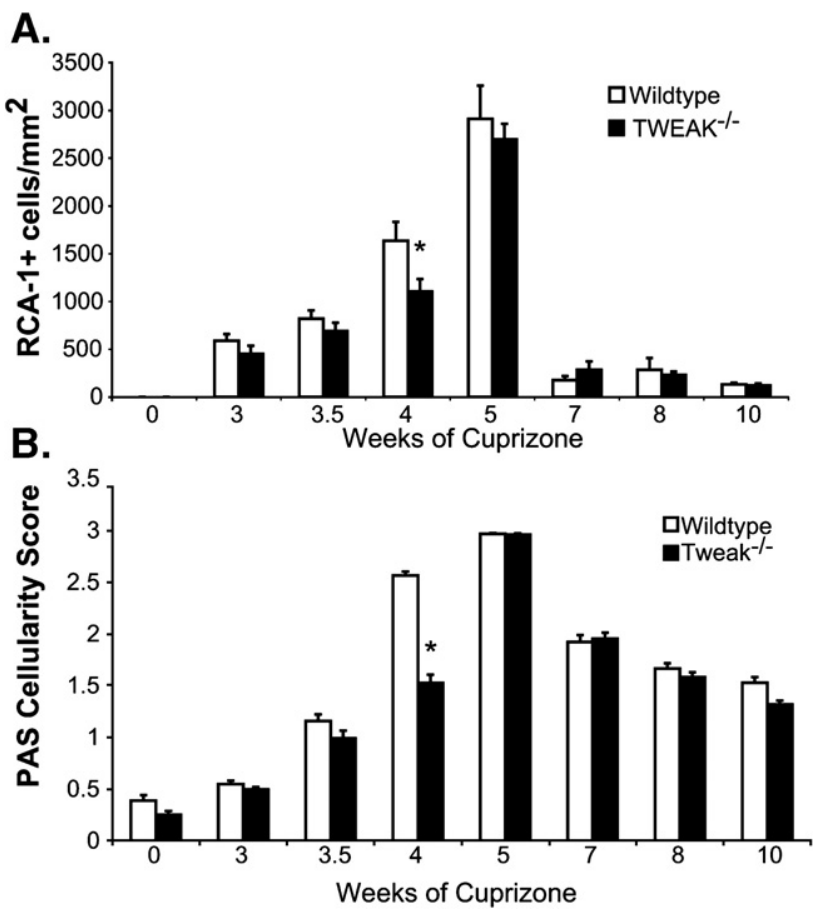

Fig. 3. Altered microglia/macrophage recruitment in $\mathrm{TWEAK}^{-1-}$ mice. Paraffin sections of wildtype and TWEAK ${ }^{-/}$mice were stained with the lectin RCA-1 and counterstained with DAPI. (A) Number of RCA-1+ cells per $\mathrm{mm}^{2}$ were calculated and represented graphically. $*$ denotes conditions in which a significant difference exists between wildtype and TWEAK ${ }^{-/-}$mice $(p=0.02)$. (B) LFB-PAS stained paraffin sections were analyzed for cellularity by 3 double-blinded readers. Scores were averaged and the results represented graphically. $*$ denotes conditions in which a significant difference exists between wildtype and TWEAK ${ }^{-/-}$mice $(p=0.002)$.

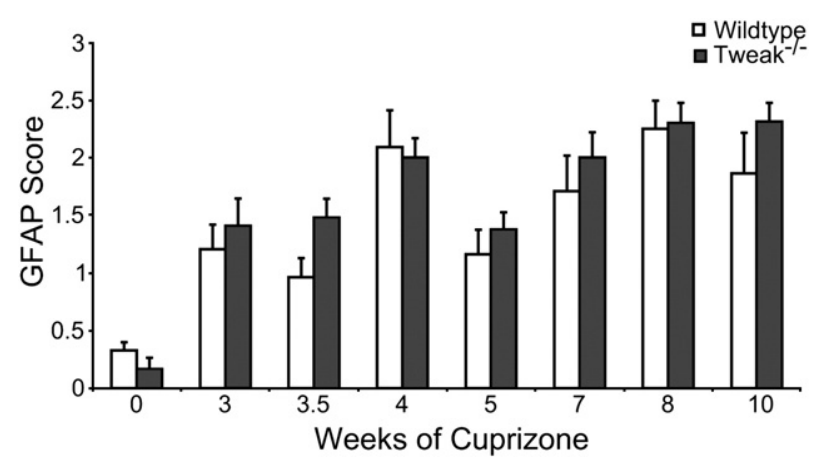

Fig. 4. Astrogliosis in wildtype and TWEAK ${ }^{-1-}$ mice. Paraffin sections of wildtype and $\mathrm{TWEAK}^{-/-}$mice were stained with antibody against glial fibrillary acidic protein and counterstained with DAPI. Sections were graded on a scale of 0 (normal) to 3 (extensive astrogliosis) and the results represented graphically. No significant differences in astrogliosis were observed between wildtype and TWEAK $^{-/-}$mice.

and macrophages. PAS scores indicate a statistically significant reduction in cellularity in $\mathrm{TWEAK}^{-/-}$mice relative to wildtype animals at the 4 week time point ( $p=0.002$ ) (Fig. 3B). This data supports the conclusions obtained from RCA-1 staining of these animals. There was no significant difference between TWEAK $^{-/-}$and wildtype microglia/macrophage accumulation or cellularity during the remyelination phase (Fig. 3). Taken together, these data indicate a role for TWEAK in enhancing neuroinflammation in the demyelination phase during cuprizone insult.

Cuprizone treatment results in astrogliosis appearing at approximately 3 weeks of treatment and sustained through 10 weeks. Evidence in the literature suggests that both TWEAK and its receptor may be expressed by astrocytes in vivo. In order to determine the role of TWEAK in astrogliosis, paraffin sections of wildtype and TWEAK ${ }^{-/-}$mice were stained for the presence of the astrocyte marker GFAP by immunohistochemistry. The extent of astrogliosis was scored on a scale of 0 (normal astrocyte number) to 3 (extensive astrogliosis). No statistically significant difference in the extent of astrogliosis was observed for any of the time points examined (Fig. 4). These data indicate that TWEAK does not function in development of astrogliosis in cuprizone treated mice.

\subsection{Effects of TWEAK on mature oligodendrocytes during demyelination}

Mature oligodendrocytes are responsible for myelination of axon fibers, thus oligodendrocyte loss frequently correlates with increased demyelination in the corpus callosum. However, because each oligodendrocyte may wrap more than one axon, these numbers need not correlate directly. To determine whether reduced or delayed oligodendrocyte loss from the corpus callosum might be responsible for the observed delay in demyelination in TWEAK ${ }^{-1}$ mice, paraffin sections of wildtype and $\mathrm{TWEAK}^{-/-}$mice were stained for the presence of the mature oligodendrocyte marker GST $\pi$ by immunohistochemistry (Fig. 5A). The number of mature oligodendrocytes in the midline corpus callosum was calculated and represented 


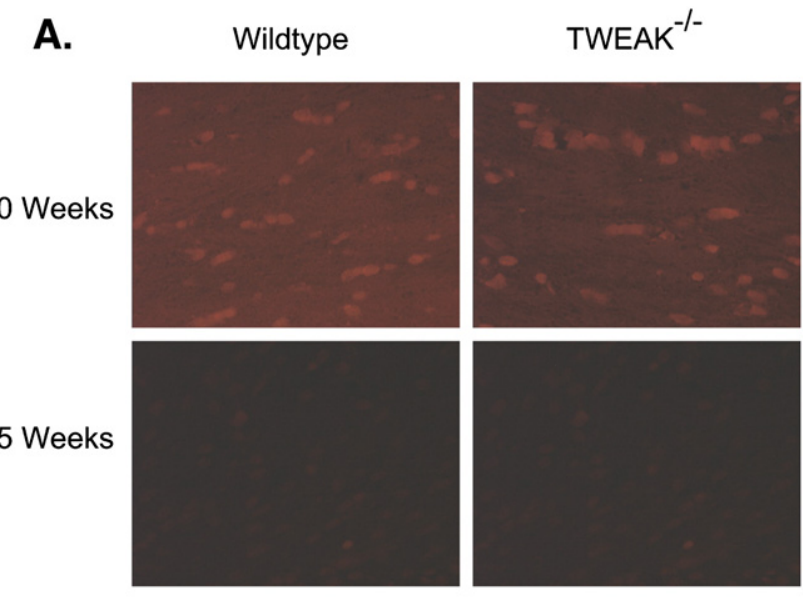

B.

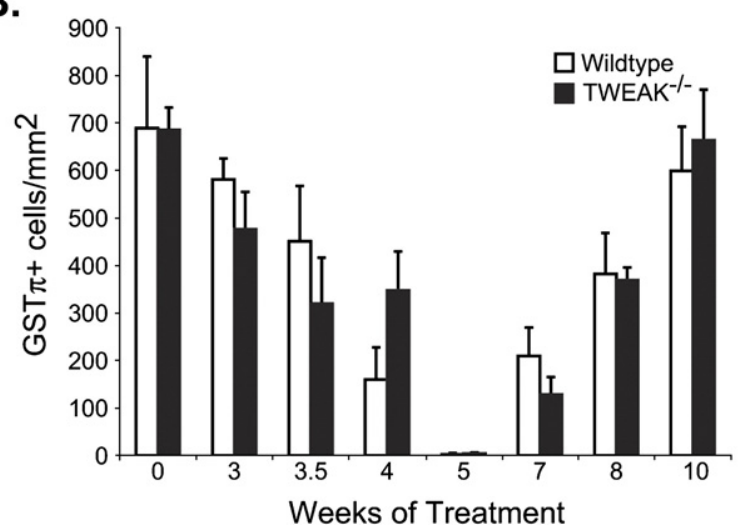

Fig. 5. Loss of mature myelinating oligodendrocytes from the corpus callosum of wildtype and $\mathrm{TWEAK}^{-/-}$mice. Paraffin sections of wildtype and TWEAK ${ }^{-/}$ mice were stained with antibody against glutathione- $S$-transferase (GST $\pi$ ). (A) Representative pictures of GST $\pi$ stained paraffin sections at 0 and 5 weeks of cuprizone treatment. (B) Number of GST $\pi+$ cells per $\mathrm{mm}^{2}$ were calculated and represented graphically.

graphically (Fig. 5B). There was a trend towards retention of greater numbers of oligodendrocytes in the corpus callosum of $\mathrm{TWEAK}^{-1-}$ mice corresponding with reduced demyelination at the 4 week time point $(p<0.06)$. It is possible that maintaining higher numbers of processes on each oligodendrocyte contributes to the retention of myelination in $\mathrm{TWEAK}^{-1-}$ mice during cuprizone treatment.

\section{Discussion}

It is important to study neuroinflammation in diverse animal models, particularly since the findings from no single model adequately recapitulate the human disease state of multiple sclerosis. The copper chelating agent cuprizone results in a predictable course of demyelination due to oligodendrocyte death and microglial infiltration, followed by remyelination after cuprizone exposure is suspended. The pathology involves areas of demyelination, large numbers of reactive astrocytes, and activated infiltrating microglia/macrophages such as is highly similar to that of type III and type IV lesions in the brains of MS patients (Lucchinetti et al., 2000). Unlike EAE, T cell infiltration in this model is rare. In further contrast to EAE, the integrity of the blood brain barrier is maintained during cuprizone treatment. Previous studies examining the role of TWEAK in EAE have demonstrated reduced clinical severity associated with reduced leukocyte infiltration in animals treated with blocking monoclonal antibodies to TWEAK (Desplat-Jego et al., 2005). However, a potential role of TWEAK within the CNS could not be ruled out. In the current study we demonstrate that a significant delay in demyelination is apparent in $\mathrm{TWEAK}^{-1}$ mice in a model system in which the blood brain barrier is not compromised and leukocyte trafficking into the CNS does not appear to be involved. Therefore, these data suggest a novel role for TWEAK in exacerbating inflammation that is operative within the CNS, and is not limited to enhancement of leukocyte recruitment to the demyelinating lesion. Pertinent to this intra-CNS function, we further show a TWEAK-dependent increase in microglia/macrophage accumulation at the site of injury, but no effect on astrogliosis during the demyelination phase. Taken together, our data support the notion that TWEAK deficiency can limit the occurrence of demyelinating lesions by limiting microglial/macrophage accumulation.

Small discrepancies exist between demyelination data obtained by LFB staining and MBP immunohistochemistry although the overall trend remains the same. There are several possible reasons for these differences. LFB stains myelin, whereas immunohistochemistry for MBP stains only one component of the myelin sheath. Furthermore, more than 14 splice forms of MBP exist and detection of all forms requires numerous antibodies. Due to the exceedingly complex nature of MBP regulation and the specificity of the antibodies used in these studies, it is not surprising that the results obtained from MBP and LFB staining differ slightly. The exacerbatory nature of TWEAK during demyelination is further supported by the trend towards the retention of greater numbers of mature oligodendrocytes in the corpus callosum of $\mathrm{TWEAK}^{-/-}$mice.

The mechanism underlying decreased microglia/macrophage accumulation in TWEAK null mice remains to be elucidated. This could be attributed to a number of mechanisms including more efficient recruitment of microglia/ macrophages, prolonged survival and/or more proliferation in the presence of TWEAK. In agreement with our findings, it was recently reported that blocking TWEAK decreased ischemia-induced microglial activation in the CNS (Yepes et al., 2005). TWEAK can be expressed by both astrocytes and microglia. TWEAK receptor, Fn14, is expressed by astrocytes (Saas et al., 2000), monocytes (Kaplan et al., 2002) and macrophages (Kim et al., 2004), though microglial expression of Fn14 has not been reported. These expression patterns suggest two possibilities that are not mutually exclusive. Toxic insult induces TWEAK production by microglia and astrocytes that directly impacts microglia/macrophages, or induces astrocyte production of cytokines, including IL-6 (Saas et al., 2000), thereby indirectly impacting microglia. Regardless of the underlying mechanism, the ability to limit lesions with pathology similar to the types III and IV lesions of MS patients suggests a promising application for TWEAK inhibition in MS. 


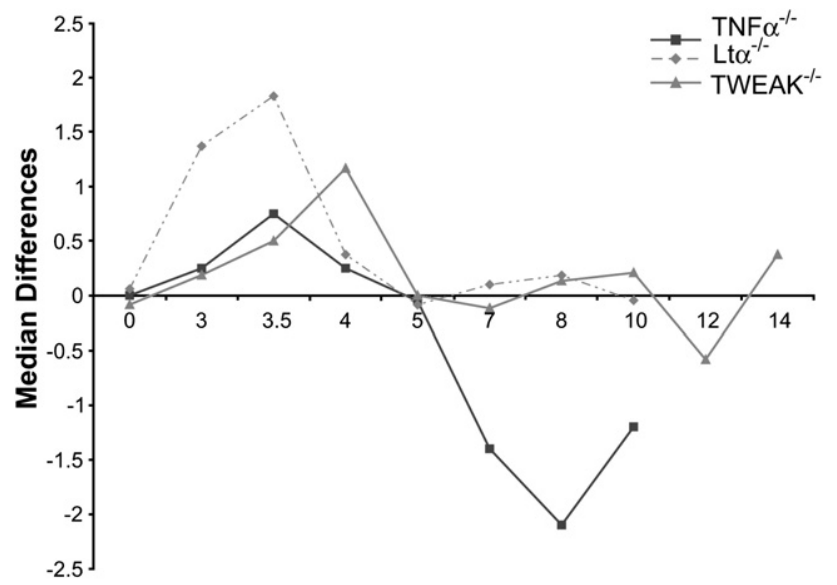

Weeks of Cuprizone

Fig. 6. Comparison of roles of TNF $\alpha, \mathrm{Lt} \alpha$ and TWEAK during demyelination and remyelination. Differences between median LFB scores of wildtype and $\mathrm{TWEAK}^{-/-}$mice were calculated as score of wildtype mice minus score of TWEAK $^{-1-}$ mice (grey line, triangles). For comparison to previously studied members of the TNF superfamily median differences between wildtype and $\mathrm{TNF}^{-/-}$mice (black line, squares) and wildtype and $\mathrm{Lt}^{-/-}$mice (dashed grey line, diamonds) are also shown. Lt $\alpha$ exhibits a dramatic exacerbatory effect on demyelination and has no effect on remyelination. TNF $\alpha$ exhibits a modest effect on demyelination and a dramatically enhances remyelination. TWEAK exerts an intermediate response on demyelination, and a small transient effect on the remyelination phase.

The time course of cuprizone treatment allows the study of both demyelination and remyelination. This is of particular importance in assessing the role of molecules that may be suitable targets for drug therapy. As an illustration, increased TNF $\alpha$ production is observed in MS, EAE and cuprizone treatment (Liu et al., 1998; Arnett et al., 2001; Kassiotis and Kollias, 2001). Thus, it would appear that TNF $\alpha$ may represent a viable therapeutic target for the treatment of demyelinating disease. However, TNF $\alpha$ inhibition in MS patients resulted in exacerbation of patient symptoms (van Oosten et al., 1996; Lenercept, 1999; Robinson et al., 2001; Sicotte and Voskuhl, 2001). Later studies examining the role of TNF $\alpha$ in remyelination revealed that TNF $\alpha$ is an essential factor in efficient repair of the CNS (Liu et al., 1998; Arnett et al., 2001; Kassiotis and Kollias, 2001). In order to directly compare the relative contributions of $\mathrm{TNF} \alpha$ and other family members we have studied to date, we compiled LFB difference scores from studies of TNF $\alpha, L t \alpha$ and TWEAK. Data are expressed as the difference in median LFB scores between cuprizone-fed knockout and wildtype mice (calculated as median LFB score of wildtype mice minus the median LFB score of knockout mice) for each study (Fig. 6). The fact that mice in the current study remyelinated somewhat more slowly than in previous studies (Arnett et al., 2001; Plant et al., 2005) indicates standard experimental variability and is the reason wildtype animals are included for direct comparison in all experiments. TNF $\alpha$ exerts a modest exacerbatory effect on demyelination. Lt $\alpha$ exerts the greatest exacerbatory effect on demyelination. TWEAK exerts a moderate exacerbatory effect on demyelination, but does so at a later time point than either $\mathrm{TNF} \alpha$ or $\mathrm{Lt} \alpha$, thus possibly suggesting a different mechanism may be responsible. Only
$\mathrm{TNF} \alpha$ markedly contributes to the remyelination process in cuprizone treated mice. Further studies are warranted to investigate whether the marginal delay in remyelination in TWEAK $^{-/}$mice is of any significant biological consequence.

In conclusion, this study represents the first report of the role of TWEAK in a microglia/macrophage-mediated model of demyelination where the blood brain barrier remains intact and the first examination of the role of TWEAK in remyelination. The delay in demyelination in TWEAK ${ }^{-/-}$mice is concomitant with a delay in microglia/macrophage infiltration and an increase in oligodendrocytes in the corpus callosum. Oligodendrocyte loss and astrogliosis proceed normally in the absence of TWEAK, and there is only a transient marginal delay in remyelination. Thus, we believe that inhibition of TWEAK may represent a promising therapeutic approach to treat MS.

\section{Acknowledgements}

This work was supported by Biogen Idec and NMSS grant RG1785 (JP-YT). HAI is an NMSS post-doctoral fellow (FG1605). BPOC is a fellow of the Irvington Institute for Immunological Research.

\section{Appendix A. Supplementary data}

Supplementary data associated with this article can be found, in the online version, at doi:10.1016/j.jneuroim.2007.12.003.

\section{References}

Akerman, P., Cote, P., Yang, S.Q., McClain, C., Nelson, S., Bagby, G.J., Diehl, A.M., 1992. Antibodies to tumor necrosis factor-alpha inhibit liver regeneration after partial hepatectomy. Am. J. Physiol. 263, G579-G585.

Arnett, H.A., Mason, J., Marino, M., Suzuki, K., Matsushima, G.K., Ting, J.P., 2001. TNF alpha promotes proliferation of oligodendrocyte progenitors and remyelination. Nat. Neurosci. 4, 1116-1122.

Baker, D., Butler, D., Scallon, B.J., O’Neill, J.K., Turk, J.L., Feldmann, M., 1994. Control of established experimental allergic encephalomyelitis by inhibition of tumor necrosis factor (activity) within the central nervous system using monoclonal antibodies and TNF receptor-immunoglobin fusion proteins. Eur. J. Immunol. 24, 2040-2048.

Black, R.A., Rauch, C.T., Kozlosky, C.J., Peschon, J.J., Slack, J.L., Wolfson, M.F., Castner, B.J., Stocking, K.L., Reddy, P., Srinivasan, S., Nelson, N., Boiani, N., Schooley, K.A., Gerhart, M., Davis, R., Fitzner, J.N., Johnson, R.S., Paxton, R.J., March, C.J., Cerretti, D.P., 1997. A metalloproteinase disintegrin that releases tumour necrosis factor-alpha from cells. Nature 385, 729-733.

Blakemore, W.F., 1972. Observations on oligodendrocyte degeneration, the resolution of status spongiosus and remyelination in cuprizone intoxication of mice. J. Neurocytol. 1, 413-426.

Blakemore, W.F., 1973a. Demyelination of the superior peduncle in the mouse induced by cuprizone. J. Neurol. Sci. 20, 63-72.

Blakemore, W.F., 1973b. Remyelination of the superior peduncle in the mouse following demyelination induced by feeding cuprizone. J. Neurol. Sci. 20, 73-83.

Brockhaus, M., Schoenfeld, H.-J., Schlaeger, E.-J., Hunziker, W., Lesslauer, W., Loetscher, H., 1990. Identification of two types of tumor necrosis factor receptors on human cell lines by monoclonal antibodies. Proc. Natl. Acad. Sci. U. S. A. 87, 3127-3131.

Brown, S.A.N., Richards, C.M., Hanscom, H.N., Feng, S.-L.Y., Winkles, J.A., 2003. The Fn14 cytoplasmic tail binds tumour-necrosis-factor-receptorassociated factors $1,2,3$, and 5 and mediates nuclear factor- $\kappa \mathrm{B}$ activation. Biochem. J. 371, 395-403. 
Brown, S.A.N., Hanscom, H.N., Vu, H., Brew, S.A., Winkles, J.A., 2006. TWEAK binding to the Fn14 cysteine-rich domain depends on charged residues located in both the A1 and D2 modules. Biochem. J. 397, 297-304.

Campbell, S., Michaelson, J., Burkly, L., Putterman, C., 2004. The role of TWEAK/Fn14 in the pathogenesis of inflammation and systemic autoimmunity. Front. Biosci. 9, 2273-2284.

Campbell, S., Burkly, L.C., Gao, H.X., Berman, J.W., Su, L., Browning, B., Zheng, T., Schiffer, L., Michaelson, J.S., Putterman, C., 2006. Proinflammatory effects of TWEAK/Fn14 interactions in glomerular mesangial cells. J. Immunol. 176, 1889-1898.

Chicheportiche, Y., Bourdon, P.R., Xu, H., Hsu, Y.-M., Scott, H., Hession, C., Garcia, I., Browning, J.L., 1997. TWEAK, a new secreted ligand in the tumor necrosis factor family that weakly induces apoptosis. J. Biol. Chem 272, 32401-32410.

Chicheportiche, Y., Fossati-Jimack, L., Moll, S., Ibnou-Zekri, N., Izui, S., 2000. Down-regulated expression of TWEAK mRNA in acute and chronic inflammatory pathologies. Biochem. Biophys. Res. Commun. 279, 162-165.

Desplat-Jego, S., Varriale, S., Creidy, R., Terra, R., Bernard, D., Khrestchatisky, M., Izui, S., Chicheportiche, Y., Boucraut, J., 2002. TWEAK is expressed by glial cells, induces astrocyte proliferation and increases EAE severity. J. Neuroimmunol. 133, 116-123 2002

Desplat-Jego, S., Creidy, R., Varriale, S., Allaire, N., Luo, Y., Bernard, D., Hahm, K., Burkly, L., Boucraut, J., 2005. Anti-TWEAK monoclonal antibodies reduce immune cell infiltration in the central nervous system and severity of experimental autoimmune encephalomyelitis. Clin. Immunol. $117,15-23$

D’Haens, G., Swijsen, C., Noman, M., Lemmens, L., Ceuppens, J., Agbahiwe, H., Geboes, K., Rutgeers, P., 2001. Etanercept in the treatment of active refractory Crohn's disease: a single-center pilot trial. Am. J. Gasteroenterol. 96, 2564-2568

Grell, M., Zimmermann, G., Gottfried, W., Chen, C.-M., Grunwalk, U., Huang, D.C.S., Lee, Y.-H.W., Durkop, H., Engelmann, H., Scheurich, P., Wajant, H., Strasser, A., 1999. Induction of cell death by tumour necrosis factor TNF receptor 2, CD40 and CD30: a role for TNFR1 activation by endogenous membrane anchored TNF. EMBO 18, 3034-3043.

Hehlgans, T., Pfeffer, K., 2005. The intriguing biology of tumour necrosis factor/tumour necrosis factor receptor superfamily: players, rules and games. Immunology $115,1-20$.

Jakubowski, A., Ambrose, C., Parr, M., Linceaum, J.M., Wang, M.Z., Zheng, T.S., Browning, B., Michaelson, J.S., Baetscher, M., Wang, B., Bissell, D.M., Burkly, L.C., 2005. TWEAK induces liver progenitor cell proliferation cell proliferation. J. Clin. Invest. 115, 2330-2340.

Kaplan, M.J., Lewis, E.E., Shelden, E.A., Somers, E., Pavlic, R., McCune, W.J., Richardson, B.C., 2002. The apoptotic ligands TRAIL, TWEAK, and Fas ligand mediate monocyte death induced by autologous lupus $\mathrm{T}$ cells. J. Immunol. 169, 6020-6029.

Kassiotis, G., Kollias, G., 2001. Uncoupling the proinflammatory from the immunosuppressive properties of tumor necrosis factor (TNF) at the p55 TNF receptor level: implications for pathogenesis and therapy of autoimmune demyelination. J. Exp. Med. 193, 427-434.

Kim, A.-H., Kang, Y.-J., Kim, W.-J., Woo, D.-K., Lee, Y., Kim, D.-I., Park, B.P., Kwon, B.S., Park, J.-E., Lee, W.-H., 2004. TWEAK can induce proinflammatory cytokines and matrix metalloproteinase-9 in macrophages. Circ. J. 68, 396-399.

Klinkert, W.E., Kojima, K., Leslauer, W., Rinner, W., Lassman, H., Wekerle, H., 1997. TNF-alpha receptor fusion protein prevents experimental autoimmune encephalomyelitis and demyelination in Lewis rats: an overview. J. Neuroimmunol. 72, 163-168.

Knight, D.M., Trinh, H., Le, J., Siegel, S., Shealy, D., McDonough, M., Scallon, B., Moore, M.A., Vilcek, J., Daddona, P., et al., 1993. Construction and initial characterization of a mouse-human chimeric anti-TNF antibody. Mol. Immunol. 30, 1443-1453.

Korner, H., Cook, M., Riminton, D.S., Lemckert, F.A., Hoek, R.M., Ledermann, B., Kontgen, F., Fazekas de St Groth, B., Sedgwick, J.D., 1997. Distinct roles for lymphotoxin-alpha and tumor necrosis factor in organogenesis and spatial organization of lymphoid tissue. Eur. J. Immunol. 27, 2600-2609.
Liu, J., Marino, M.W., Wong, G., Grail, D., Dunn, A., Bettadapura, J., Slavin, A.J., Old, L., Bernard, C.C., 1998. TNF is a potent anti-inflammatory cytokine in autoimmune-mediated demyelination. Nat. Med. 4, 78-83.

Lucchinetti, C., Bruck, W., Parisi, J., Scheithauer, B., Rodriguez, M., Lassman, H., 2000. Heterogeneity of multiple sclerosis lesions: implications for the pathogenesis of demyelination. Ann. Neurol. 47, 707-717.

Matusevicius, D., Navikas, V., Soderstrom, M., Xiao, B.G., Haglund, M., Fredrikson, S., Link, H., 1996. Multiple sclerosis: the proinflammatory cytokines lymphotoxin-alpha and tumor necrosis factor-alpha and upregulated in cerebrospinal fluid mononuclear cells. J. Neuroimmunol. 66, 115-123.

McMahon, E.J., Suzuki, K., Matsushima, G.K., 2002. Peripheral macrophage recruitment in cuprizone-induced CNS demyelination despite an intact blood brain barrier. J. Neuroimmunol. 130, 32-45.

Michaelson, J.S., Cho, S., Browning, B., Zheng, T.S., Lincecum, J.M., Wang, M.Z., Hsu, Y.M., Burkly, L.C., 2005. TWEAK induces mammary epithelial branching morphogenesis. Oncogene 24, 2613-2624.

Mohler, K.M., Torrance, D.S., Smith, C.A., Goodwin, R.D., Stremler, K.E., Fung, V.P., Madani, H., Widmer, M.B., 1993. Soluble tumor necrosis factor (TNF) receptors are effective therapeutic agents in lethal endotoxemia and function simultaneously as both TNF carriers and TNF antagonists. J. Immunol. 151, 1548-1561.

Nakayama, M., Ishidoh, K., Kayagaki, N., Kojima, Y., Yamaguchi, N., Nakano, H., Kominami, E., Okumura, K., Yagita, H., 2002. Multiple pathways of TWEAK-induced cell death. J. Immunol. 168, 734-743.

Nakayama, M., Ishidoh, K., Kojima, Y., Harada, N., Kominami, E., Okumura, K., Yagita, H., 2003. Fibroblast growth factor-inducible 14 mediates multiple pathways of TWEAK-induced cell death. J. Immunol. 170, 341-348.

Navikas, V., He, B., Link, J., Haglund, M., Soderstrom, M., Fredrikson, S., Ljungdahl, A., Hojeberg, J., Qiao, J., Olsson, T., et al., 1996. Augmented expression of tumour necrosis factor-alpha and lymphotoxin in mononuclear cells in multiple sclerosis and optic neuritis. Brain 119, 213-223.

Perper, S.J., Browning, B., Burkly, L.C., Weng, S., Gao, C., Giza, K., Su, L., Tarilonte, L., Crowell, T., Rajman, L., Runkel, L., Scott, M., Atkins, G.J., Findlay, D.M., Zheng, T.S., Hess, H., 2006. TWEAK is novel arthritogenic mediator. J. Immunol. 177, 2610-2620.

Plant, S.R., Arnett, H.A., Ting, J.P., 2005. Astroglial-derived lymphotoxin-alpha exacerbates inflammation and demyelination, but not remyelination. Glia $49,1-14$

Plant, S.R., Wang, Y., Vasseur, S., Thrash, J.C., McMahon, E.J., Bergstrahl, D.T., Arnett, H.A., Miller, S.D., Carson, M.J., Iovanna, J.L., Ting, J.P., 2006. Upregulation of the stress-associated gene $\mathrm{p} 8$ in mouse models of demyelination and in multiple sclerosis tissues. Glia 53, 529-537.

Plant, S.R., Iocca, H.A., Wang, Y., Thrash, J.C., O'Connor, B.P., Arnett, H.A., Fu, Y.X., Carson, M.J., Ting, J.P., 2007. Lymphotoxin beta receptor: dual roles in demyelination and remyelination and successful therapeutic intervention using Lt $\beta$ R-Ig protein. J. Neurosci. 27, 7429-7437.

Probert, L., Eugster, H.P., Akassoglou, K., Bauer, J., Frei, K., Lassman, H., Fontana, A., 2000. TNFR1 signaling is critical for the development of demyelination and the limitation of T-cell responses during immunemediated CNS disease. Brain 123, 2005-2019.

Robinson, W.H., Genovese, M.C., Moreland, L.W., 2001. Demyelinating and neurologic evens reported in association with tumor necrosis factor alpha antagonism: by what mechanisms could tumor necrosis factor alpha antagonists improve rheumatoid arthritis but exacerbate multiple sclerosis? Arthritis Rheumatol. 44, 1977-1983.

Ruddle, N.H., Bergman, C.M., McGrath, K.M., Lingenheld, E.G., Grunnet, M.L., Padula, S.J., Clark, R.B., 1990. An antibody to lymphotoxin and tumor necrosis factor prevents transfer of experimental allergic encephalomyelitis. J. Exp. Med. 172, 1193-1200.

Ruuls, S.R., Hoek, R.M., Ngo, V.N., McNeil, T., Lucian, L.A., Janatpour, M.J., Korner, H., Scheerens, H., Hessel, E.M., Cyster, J.G., McEvoy, L.M., Sedgwick, J.D., 2001. Membrane-bound TNF supports secondary lympoid organ structure but is subservient to secreted TNF in driving autoimmune inflammation. Immunity $15,533-543$.

Saas, P., Boucraut, J., Walker, P.R., Quiquerez, A.L., Billot, M., Desplt-Jego, S., Chicheportiche, Y., Dietrich, P.Y., 2000. TWEAK stimulation of astrocytes and proinflammatory consequences. Glia 32, 102-107. 
Sicotte, N.L., Voskuhl, R.R., 2001. Onset of multiple sclerosis associated with anti-TNF therapy. Neurol. 57, 1885-1888.

Streit, W.J., Semple-Rowland, S.L., Hurley, S.D., Miller, R.C., Popovich, P.G., Stokes, B.T., 1998. Cytokine mRNA profiles in contused spinal cord and axotomized facial nucleus suggest a beneficial role for inflammation and gliosis. Exp. Neurol. 152, 74-87.

Suzuki, K., Kikkawa, Y., 1969. Status spongiosus of CNS and hepatic changes induced by cuprizone (biscyclohexanone oxalydihydrazone). Am. J. Pathol. 54, 307-325.

Targan, S.R., Hanauer, S.B., van Deventer, S.J., Mayer, L., Present, D.H., Braakman, T., DeWoody, K.L., Schaible, T.F., Rutgeerts, P.J., 1997. A shortterm study of chimeric monoclonal antibody cA2 to tumor necrosis factor $\alpha$ for Crohn's disease. Crohn's Disease cA2 Study Group. New Engl. J. Med. 337, 1029-1035.

TNF neutralization in MS: results of a randomized, placebo-controlled multicenter study. The Lenercept Multiple Sclerosis Study Group and The University of British Columbia MS/MRI Analysis Group. 1999. Neurology 53, 457-465. van Oosten, B.W., Barkhof, F., Truyen, L., Boringa, J.B., Bertelsmann, F.W., von Blomberg, B.M., Woody, J.N., Hartung, H.P., Polman, C.H., 1996. Increased MRI activity and immune activation in two multiple sclerosis patients treated with the monoclonal anti-tumor necrosis factor antibody cA2. Neurology 47, 1531-1534.

Wiley, S.R., Winkles, J.A., 2003. TWEAK, a member of the TNF superfamily, is a multifunctional cytokine that binds the TWEAKR/Fn14 receptor. Cytokine Growth Factor Rev. 14, 241-249.

Woodroofe, M.N., Cuzner, M.L., 1993. Cytokine mRNA expression in inflammatory multiple sclerosis lesions: detection by non-radioactive in situ hybridization. Cytokine 5, 583-588.

Yepes, M., Brown, S.A., Moore, E.G., Smith, E.P., Lawrence, D.A., Winkles, J.A., 2005. A soluble Fn14-Fc decoy receptor reduces infarct volume in a murine model of cerebral ischemia. Am. J. Pathol. 166, 511-520. 